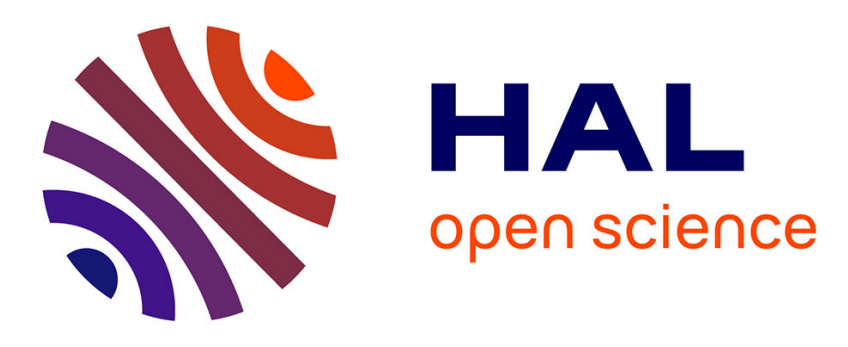

\title{
Adaptive Path Planning for Steerable Needles Using Duty-Cycling
}

Mariana Costa Bernardes, Bruno Vilhena Adorno, Philippe Poignet, Nabil Zemiti, Geovany A. Borges

\section{- To cite this version:}

Mariana Costa Bernardes, Bruno Vilhena Adorno, Philippe Poignet, Nabil Zemiti, Geovany A. Borges. Adaptive Path Planning for Steerable Needles Using Duty-Cycling. IROS: Intelligent RObots and Systems, Sep 2011, San Francisco, CA, United States. pp.2545-2550, 10.1109/IROS.2011.6048259 . lirmm-00641824

\section{HAL Id: lirmm-00641824 https://hal-lirmm.ccsd.cnrs.fr/lirmm-00641824}

Submitted on 16 Nov 2011

HAL is a multi-disciplinary open access archive for the deposit and dissemination of scientific research documents, whether they are published or not. The documents may come from teaching and research institutions in France or abroad, or from public or private research centers.
L'archive ouverte pluridisciplinaire HAL, est destinée au dépôt et à la diffusion de documents scientifiques de niveau recherche, publiés ou non, émanant des établissements d'enseignement et de recherche français ou étrangers, des laboratoires publics ou privés. 


\title{
Adaptive path planning for steerable needles using duty-cycling
}

\author{
Mariana C. Bernardes, Bruno V. Adorno, Philippe Poignet, Nabil Zemiti, Geovany A. Borges
}

\begin{abstract}
This paper presents an adaptive approach for 2D motion planning of steerable needles. It combines dutycycled rotation of the needle with the classic Rapidly-Exploring Random Tree (RRT) algorithm to obtain fast calculation of feasible trajectories. The motion planning is used intraoperatively at each cycle to compensate for system uncertainties and perturbations. Simulation results demonstrate the performance of the proposed motion planner on a workspace based on ultrasound images.
\end{abstract}

\section{INTRODUCTION}

Percutaneous medical procedures usually involve the insertion of a needle deep into soft tissue and depend on precise tip positioning for effectiveness. Needle deflection and tissue deformation are the most important factors that affect needle insertion accuracy and require great expertise from the surgeon to compensate for their effects. In addition, the procedure target may be located in a region of difficult access, which cannot be reached by conventional rigid needles without causing excessive, injurious pressure on tissue. This situation is critical in the presence of obstacles in the needle path, such as important organs or vessels.

Special needles capable of active steering during its insertion have been designed to overcome such problems and also expand the applicability of percutaneous procedures [1]. These needles use their great flexibility and beveled tips to enhance and magnify the needle deflection effect, allowing curved trajectories that could be used to avoid sensitive or impenetrable areas inaccessible with the traditional technique.

When inserted into tissue, a steerable needle follows a path that is prescribed by the geometry of its beveled tip, its relative stiffness with respect to the tissue and the insertion and twist velocities at the needle entry point. In robotics, this type of needle can be described as a kinematic system with nonholonomic constraints. As a consequence, motion planning is a complex task and its difficulty increases as we consider the presence of uncertainties due to errors in tip positioning, needle modeling, tissue inhomogeneity and deformation. Thus, the need of developing a robotic steering system capable of compensating for such effects.

Medical imaging can be used not only for the planning but also for the control of robot-assisted medical procedures. The use of $2 \mathrm{D}$ ultrasound imaging is specially attractive because it is safe, affordable and provides information related to

M.C. Bernardes, B.V. Adorno, P. Poignet and N. Zemiti are with Université Montpellier 2, LIRMM, 161 rue Ada, 34095 Montpellier, France \{bernardes, adorno, poignet, zemiti\}@lirmm. fr

G.A. Borges and M.C. Bernardes are with Universidade de Brasília, LARA, Caixa Postal 4386, Zip Code 70919-970, Brasília - DF, Brazil \{gaborges, bernardes\} @unb.br

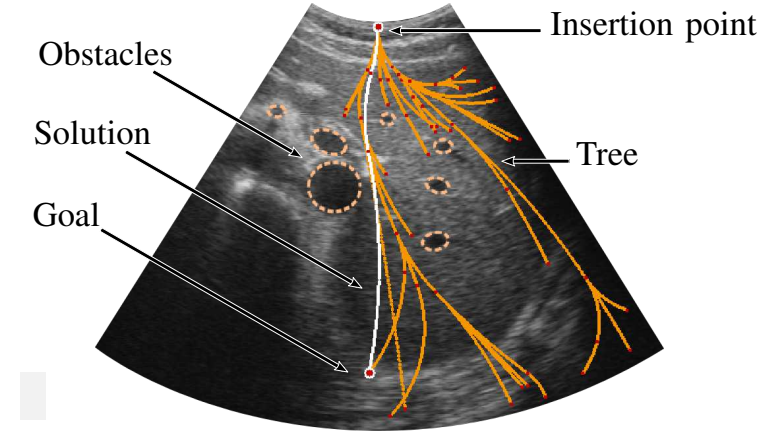

Fig. 1: A typical tree built by the Arc-based RRT to search for a feasible path and its corresponding solution.

tissue properties, target displacement and tool position [2]. For using such type of medical imaging in robotic needle steering, it is desirable to have a fast method for $2 \mathrm{D}$ path planning that respects the system nonholonomic constraints.

In this paper, we present a new method that uses dutycycled rotation of the needle combined with the classic Rapidly-Exploring Random Tree (RRT) algorithm to obtain fast calculation of feasible trajectories and we evaluate its performance in a simulated insertion procedure with simultaneous replanning.

\section{RELATED WORK}

Since the end of the '90s, several motion planners capable of tackling nonholonomic constraints have been proposed. Many of these approaches rely on sampling-based techniques, like the Probabilistic Roadmaps [3] or the RapidlyExploring Random Tree [4]. The main advantage of the RRT method is the exemption of a pre-processing phase, in addition to being very fast, easy to implement and probabilistic complete [5].

Many path planning methods use the steerable needle nonholonomic kinematics for finding feasible paths. Park et al. [6] proposed a diffusion based approach, but they only considered obstacle-free 3D environments. Duindam et al. [7] used explicit geometric inverse kinematics for 2D and 3D needle motion planning and $\mathrm{Xu}$ et al. [8] were the first to apply RRT-based methods to steerable needle motion planning.

However, only a few planning methods deal with needle motion uncertainty caused by tissue deformation and interaction forces. Alterovitz et al. [9] used a finite element mesh to compute soft tissue deformations combined to numerical optimization to find a locally optimal initial configuration and insertion distance. A finite element method has also been 
used by Vancamberg et al. [10] to minimize the final error of a RRT solution in a breast biopsy application. But the efficiency of these strategies depends a lot on the quality of the mesh simulation and how accurately it represents the real tissue.

Instead of simulating a tissue mesh, Alterovitz et al. considered uncertainty in needle motion by formulating the planning problem as a Markov Decision Process, using a discretization of the state space [11] and using a Stochastic Roadmap [12]. These methods presented great results for preoperative planning but are not suitable for an adaptive intraoperative system due to its extensive precomputations. Hauser et al. [13] were the only to propose a control-loop policy to deal with uncertainties during motion planning and control. But again, only obstacle-free environments were considered.

\section{A. Contributions and organization of the paper}

The main contribution of this work is the proposal of an adaptive strategy that applies fast replanning during the insertion procedure to compensate for system uncertainties, like tissue deformation, tissue inhomogeneity, positioning errors and other modeling approximations. Also, we developed a new Arc-based RRT path planner for steerable needles using a duty-cycle strategy for insertion (Fig. 1). It uses explicit geometry to obtain feasible trajectories that respect the needle nonholonomic constraints, allowing the planner to be used intraoperatively due to its high success rate and fast calculation.

This paper is organized as follows. Section III describes the steerable needle nonholonomic model and the principle of the duty-cycle insertion strategy. Then, in Section IV the Arcbased RRT planner is detailed, with a brief description of the path planning problem and the proposed algorithm. Section $\mathrm{V}$ presents the replanning strategy while in Section VI we present the results obtained and the evaluation of our method. Finally, in Section VII we discuss its use possibilities, next steps in development and future works.

\section{Steerable NeEdLE Kinematic MOdel}

When pushed forward, the natural behavior of a steerable needle is to bend in the direction of its sharpened tip, following an arc of approximately constant curvature $\kappa_{\max }$. The kinematic model for this kind of needle can be approximated by that of a nonholonomic unicycle vehicle [1], with the following nonholonomic constraints: $\omega_{y}=\nu_{y}=\nu_{z}=0$ and $\omega_{z}=\nu_{x} \kappa_{\max }$. Thus, the system has two control inputs $\nu_{x}$ and $\omega_{x}$, that are respectively the needle's insertion and rotation velocities along its shaft, and are referred simply as $\nu$ and $\omega$ (Fig. 2).

The usual technique for needle steering in a $2 \mathrm{D}$ plan, called stop-and-turn [14], uses pure insertion of the needle combined with occasional $180^{\circ}$ rotations to reorient the bevel whenever there is a curvature inversion. In consequence, the needle can only reach points that belong to arcs of constant curvature, limiting path possibilities. This may result in a slow planning, normally not suitable for intraoperative use.

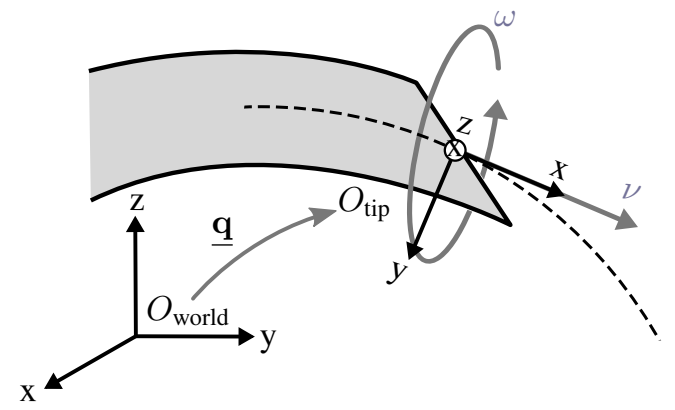

Fig. 2: Needle tip 3D representation and system inputs.

However, if we combine simultaneous rotation and insertion velocities, the needle moves along an helical path in the 3D space. In the case of using a rotation velocity relatively larger than the insertion velocity, the helix curvature tends to zero. Consequently, the needle follows a straight trajectory in its $x y$-plane. The duty-cycle strategy [15] explores such idea to achieve different curvature values.

This strategy combines periods $T_{\text {ins }}$ of pure insertion with periods $T_{\text {rot }}$ of simultaneous insertion and rotation, so that any curvature ranging from the natural curvature to a pure straight trajectory can be achieved. When considering the duty-cycle strategy to insert the needle along a 2D plan, we simplify the path planning problem to that of a car-like mobile robot that is subjected to two nonholonomic constraints: it can only move with continuous tangent direction and its turning radius is lower bounded. Also, the use of rotational motion has been proved to reduce the amount of tissue indentation as well as the friction force between the needle shaft and the tissue [16].

The duty-cycle $D C$ is defined as the ratio of the rotation period to the cycle period $T$ :

$$
D C=\frac{T_{\mathrm{rot}}}{T},
$$

where $T=T_{\text {rot }}+T_{\text {ins }}$.

There is a linear relationship between curvature and dutycycle [17]. So any path curvature in the range $\left[0, \kappa_{\max }\right]$ can be obtained by a proper choice of $D C$ :

$$
\kappa=\kappa_{\text {max }}(1-D C),
$$

where $\kappa$ is the effective curvature and $\kappa_{\max }$ is the needle natural curvature, when no spinning is applied.

To keep the needle in the working 2D plane, the rotation speed is chosen to be much higher than the insertion speed. Also, the needle must perform complete rotations at each cycle. Consequently, the rotation period is chosen to be $2 \pi n \omega^{-1}$ with $n=1,2,3, \ldots$. By making $T_{\text {rot }}$ a fixed value, the insertion period $T_{\text {ins }}$ should be adjusted in order to achieve a desired curvature. This method can be easily implemented by fixing the rotational speed $\omega$ and moving a fixed insertion distance $\triangle s$ at each cycle, so that the insertion velocity is variable and given by $\nu=\triangle s / T$. The coordination between rotation and insertion motion is considered achieved by the use of a controller, out of the scope of this paper. 
The 3D configuration of the needle tip can be described by a rigid transformation from $O_{\text {world }}$ to $O_{\text {tip }}$ in dual quaternion representation (Fig. 2). In this notation, the rigid transformation is a combination of a translation $\vec{t}=[x, y, z]$ from the origin of $O_{\text {world }}$ to $O_{\text {tip }}$, followed by a rotation of an angle $\phi$ about an axis $\vec{n}=\left[n_{x}, n_{y}, n_{z}\right]$ through the origin of $O_{\text {tip }}$. Consequently, the tip configuration $\underline{\mathbf{q}}$ is given by

$$
\underline{\mathbf{q}}=\left[\begin{array}{cc}
\mathbf{q} & \frac{1}{2} \mathbf{t q}
\end{array}\right],
$$

where $\mathbf{q}=\left[\cos \left(\frac{\phi}{2}\right), \sin \left(\frac{\phi}{2}\right) \vec{n}\right]$ and $\mathbf{t}=[0, \vec{t}]$ are the respective rotation and translation quaternions of the needle tip frame with respect to the world frame [18].

A discrete implementation of the needle kinematic model can be obtained by a combination of consecutive dual quaternion multiplications

$$
\underline{\mathbf{q}}_{k+1}=\underline{\mathbf{q}}_{k} \underline{\mathbf{q}}_{\mathrm{rot}} \underline{\mathbf{q}}_{\mathrm{ins}},
$$

where $\underline{\mathbf{q}}_{\mathrm{rot}}$ represents the movement during simultaneous rotation and insertion, and $\underline{\mathbf{q}}_{\mathrm{ins}}$ is the movement of the insertion-only period.

Consequently, we have

$$
\underline{\mathbf{q}}_{\mathrm{rot}}=\left[\begin{array}{ll}
\mathbf{q}_{\mathrm{rot}} & \frac{1}{2} \mathbf{t}_{\mathrm{rot}} \mathbf{q}_{\mathrm{rot}}
\end{array}\right] \text { and } \underline{\mathbf{q}}_{\mathrm{ins}}=\left[\begin{array}{ll}
\mathbf{q}_{\mathrm{ins}} & \frac{1}{2} \mathbf{t}_{\mathrm{ins}} \mathbf{q}_{\mathrm{ins}}
\end{array}\right] \text {, }
$$

where

$$
\begin{aligned}
\mathbf{q}_{\mathrm{rot}} & =\left[\cos \left(\frac{\phi_{\mathrm{rot}}}{2}\right), \sin \left(\frac{\phi_{\mathrm{rot}}}{2}\right) \frac{\vec{n}}{\|\vec{n}\|}\right], \quad \vec{n}=\left[\omega, 0, \nu \kappa_{\mathrm{max}}\right] \\
\mathbf{t}_{\mathrm{rot}} & =\left[0, \nu T_{\mathrm{rot}}, 0,0\right], \quad \phi_{\mathrm{rot}}=\|\vec{n}\| T_{\mathrm{rot}} \\
\mathbf{q}_{\text {ins }} & =\left[\cos \left(\frac{\phi_{\text {ins }}}{2}\right), 0,0, \sin \left(\frac{\phi_{\text {ins }}}{2}\right)\right] \\
\mathbf{t}_{\text {ins }} & =\left[0, \nu T_{\mathrm{ins}}, 0,0\right], \quad \phi_{\mathrm{ins}}=\nu \kappa_{\mathrm{max}} T_{\mathrm{ins}}
\end{aligned}
$$

\section{DUty-CYCLING MOTION PLANNING}

Although the kinematic model defines the needle tip configuration in $3 \mathrm{D}$ space, we want to move the needle only in the ultrasound plane. Thus, for planning purposes we assume that the needle $x y$-plane is correctly aligned to the desired 2D plane of the medical images and we let the configuration $q$ of a needle to be defined by its tip cartesian coordinates $p=(x, y)^{T}$ and orientation angle $\theta$.

The objective of the planner is to find a combination of circular arcs capable of taking the needle from its initial configuration $q_{\text {init }}=\left(x_{\text {init }}, y_{\text {init }}, \theta_{\text {init }}\right)^{T}$ to a final position $p_{\text {goal }}=\left(x_{\text {goal }}, y_{\text {goal }}\right)^{T}$ while respecting the nonholonomic constraints. An arc $\mathcal{A}$ is defined by its curvature $\kappa$ and its two end configurations $q_{A}=\left(x_{A}, y_{A}, \theta_{A}\right)^{T}$ and $q_{B}=$ $\left(x_{B}, y_{B}, \theta_{B}\right)^{T}$. The final extremity of each arc should correspond to the next arc's initial extremity, not only in position but also in orientation, so we have $C^{1}$ continuity. The goal orientation is not considered as a problem requirement since in a percutaneous procedure the final needle orientation is usually irrelevant. Thus, $\theta_{\text {goal }}$ is used as an extra degree of freedom to obtain such orientation continuity. The workspace is a $2 \mathrm{D}$ plane with boundaries defined to be the ultrasound image area, and the locations of the targets and obstacles are considered known and defined by the surgeon.

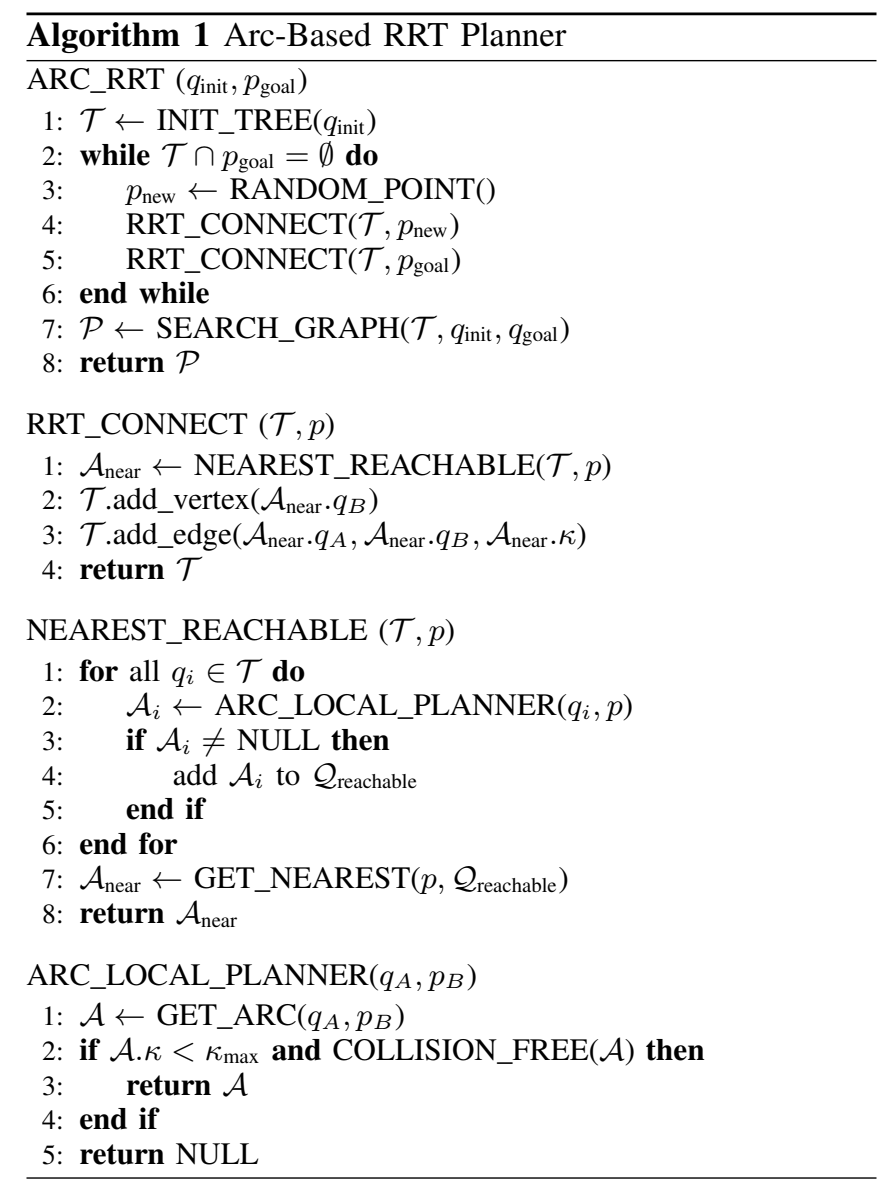

\section{A. Arc-Based RRT}

The pseudocode for our planner is shown in Algorithm 1 and was based on the classic RRT approach [4]. First, a tree $\mathcal{T}$ rooted in $q_{\text {init }}$ is constructed. For a point $p_{\text {new }}$ randomly sampled from the free space, we define the set $\mathcal{Q}_{\text {reachable }}$ of all nodes in $\mathcal{T}$ from which $p_{\text {new }}$ can be reached. To respect the needle constraints, we propose a geometric-based Arc Local Planner that calculates the only arc capable of connecting two points given that the first point is associated to a known orientation. If the obtained arc respects the curvature range and does not intersect any obstacle, it is considered reachable. Then, the nearest arc in $\mathcal{Q}_{\text {reachable }}$ is added to $\mathcal{T}$. The tree is expanded until it can be connected to the target by the local planner or until a maximum number of nodes is reached. Then, a graph search is conducted to return the obtained path $\mathcal{P}$.

\section{B. Arc Local Planner}

The local planner proposed is responsible for calculating the arc's curvature and final orientation given an initial configuration $q_{A}$ and a final point $p_{B}$. This can be done geometrically with only a few trigonometric calculations as discussed bellow.

Consider two 2D points $A$ and $B$, and an initial orientation in $A$, given by $\theta_{A}$ (Fig. 3). First, we calculate the distance $d$ from point $A$ to point $B$ and the signed bearing angle $\varphi$

$$
\varphi=\arctan \left(y_{B}-y_{A}, x_{B}-x_{A}\right)-\theta_{A} .
$$




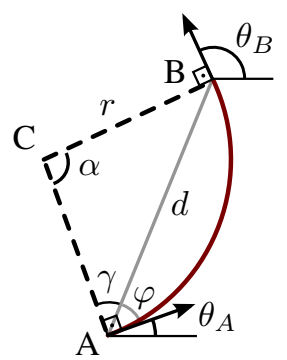

Fig. 3: Arc connecting points A and B.

Note that angles are represented in the interval $(-\pi, \pi]$.

From Fig. 3, we can see that $\varphi+\gamma=\frac{\pi}{2}$ and $\alpha+2 \gamma=\pi$, so we have that $\alpha=2 \varphi$. Using the Law of Sines and the relationship between the arc and the bearing angle, we find that the arc curvature is given by

$$
\kappa=\frac{1}{r}=\frac{2 \sin (\varphi)}{d} .
$$

The orientation in point $\mathrm{B}$ is $\theta_{B}=\alpha+\theta_{A}$.

Finally, the resultant arc is $\mathcal{A}=\left[q_{A}, q_{B}, \kappa\right]$. The arc is accepted by the Arc Local Planner if $\kappa$ respects the maximum curvature constraint and if the arc path is collision free. Otherwise, the point $p_{B}$ is considered unreachable from the configuration $q_{A}$.

\section{ADAPTIVE REPLANNING}

From the sequence of arcs provided by the Arc-Based RRT planner and using the duty-cycle equations, we obtain the sequence of duty-cycle inputs that will take the needle from its insertion point to the goal while following the desired path. However, tissue deformation and inhomogeneity, imprecision of the unicycle model, and uncertainties in needle and obstacles positions may deviate the needle from the 2D working plane or from the planned trajectory, leading to a possible collision with an important organ or misplacement of the needle tip at the end of the insertion task

The problem of keeping the needle in the 2D plane has already been studied and a low-level controller was proposed to stabilize the needle in a given plane [19]. For the remaining problem of trajectory deviation, we propose a replanning strategy to systematically correct the needle path in order to avoid collisions and converge to the final target.

Our adaptive replanning is executed every cycle until the needle tip is sufficiently close to the target. Its pseudocode is shown in Algorithm 2. We assume that current information about the workspace is provided by an ultrasound tracking system which is out of the scope of this paper. The ultrasound feedback is used to update the path by considering the needle's current configuration as the new $q_{\text {init }}$ and the target's current position as the new $p_{\text {goal }}$. Then, the Arc Local Planner is run to adjust all arcs from $\mathcal{P}$, recalculating the new curvatures and final orientations. If a collision is detected in the updated arcs, or if the new curvature does not respect the maximum limit, the complete RRT planner is run again to find a new feasible trajectory.

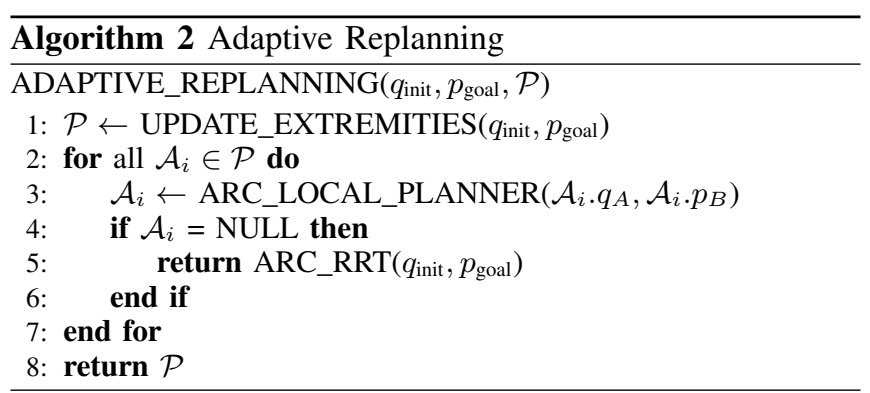

The Arc Local Planner is extremely fast since it uses explicit geometry to satisfy the system nonholonomic constraints. We performed timing experiments on a $3 \mathrm{GHz} \mathrm{PC}$ and the average execution time for executing ARC_LOCAL_PLANNER was $0.038 \mathrm{~ms}$ in 10000 trials, with standard deviation of $0.033 \mathrm{~ms}$. Also, it presents low rejection of samples since we have an extra degree of freedom in the choice of the final orientations. Consequently, the Arcbased RRT is able to return a solution from a small quantity of nodes, which normally represents only a few milliseconds of processing. Considering that needle insertion procedures normally occur at small insertion and rotation velocities due to safety restrictions, the adaptive replanning can be easily used intraoperatively.

Even though the convergence of our method is not assured, the RRT is proved to be probabilistic complete, meaning that if we give it enough time to search for the solution and if the solution exists, it will be found [5]. In practice, what we observe is that the Arc-based RRT converges much before the next cycle due to its high success rate and fast execution when compared to the insertion procedure speed.

Also, the resultant path is not the shortest possible since we do not use any expensive numerical optimization in favor of intraoperative use. Nevertheless, the general quality of the solutions converges to the optimum as we increase the number of executions of the Arc-based RRT. Consequently, if the adaptive replanner profits from the time left between cycles to accumulate executions, the quality of the path will get close to optimal.

\section{RESULTS}

\section{A. Path Planning Evaluation}

We tested the proposed Arc-Based RRT planner for needle steering in the $2 \mathrm{D}$ environment with obstacles depicted in Fig. 1. The workspace is based on an abdominal ultrasound image obtained with a General Electric Diasonics Synergy equipment and is defined to be the area covered by the ultrasound transducer and the obstacles are seven round structures distinguishable in the image. For the kinematic model of the needle, we specified a needle radius of curvature of $6.01 \mathrm{~cm}$. Simulations are run in a PC with Intel Core 2 Duo $3.00 \mathrm{GHz}$, 3.2 GB memory and Ubuntu 10.04 operational system. To validate the proposed planner, we performed two different simulations.

1) Simulation 1: The first simulation evaluates the capability of the proposed algorithm to find a solution. For each 
TABLE I: Arc-Based RRT performance in Simulation 1.

\begin{tabular}{lc}
\hline Number of trials & 10000 \\
Number of successes & 10000 \\
Average number of nodes & 114 \\
Average CPU time (ms) & 3.05 \\
\hline
\end{tabular}

trial, an initial configuration and a goal point are randomly picked from an uniform distribution. The maximum number of nodes for the RRT is 2500 . Table I presents the results of Simulation 1 for 10000 trials. Feasible paths were found in all cases, with an average of 114 nodes, which corresponds to an average of 3.05 milliseconds of CPU time used.

2) Simulation 2: The second simulation evaluates the quality of the paths generated. For this, we defined the average path length as our quality criterion. The initial configuration and the target point are the same for all tests in Simulation 2 and were arbitrarily chosen in the image as $q_{\text {init }}=\left[323,92, \frac{\pi}{2}\right]$ and $p_{\text {goal }}=[315,412]$ given in pixels and radians. The optimal path length for this configuration is $120.15 \mathrm{~mm}$. The maximum number of nodes allowed for the RRT is 2500 .

For Simulation 2, we also had $100 \%$ of success after 10000 trials. However, the average path length was $32 \%$ higher than the optimal, with a standard deviation of $28.10 \mathrm{~mm}$. The smallest path from all trials was $120.15 \mathrm{~mm}$ while the longest one was $410.19 \mathrm{~mm}$ (see results for 1 RRT in Fig. 4).

To overcome such high variance in the path length, we proposed using extra CPU time to assure a good quality path. The idea is to build more trees to explore the workspace independently, and then, to pick the solution with the shortest path. The extra time used to compute the path is not harmful to the overall performance of our system since the dynamic of the needle insertion is very slow when compared to the RRT exploration which is in the range of a few milliseconds.

Fig. 4 summarizes the results for tests with 1, 10, 20 and 50 simultaneous trees. For all cases we had the same 100\% success rate, but very distinct results for the average path length. The results show that despite the increase in CPU time, the addition of more RRT's to explore the workspace is justified by the improvement in path quality and smaller variance.

\section{B. Needle Insertion Simulation ${ }^{1}$}

1) Simulation 3: The third simulation evaluates if the nonholonomic constraints are respected and if the needle is capable of following the expected trajectory during an open-loop insertion procedure. For this, the needle tip was simulated using the discrete model from (5). The rotational velocity was set to $2 \mathrm{~Hz}$ and $T_{\text {rot }}=0.5 \mathrm{~s}$. The insertion distance at each cycle was defined to be $\triangle s=1 \mathrm{~mm}$. The workspace is the same used in previous simulations.

We used the Arc-based RRT to obtain a feasible path and the correspondent sequence of duty-cycles parametrized in path length was directly calculated from the arc's curvatures and (2). The sequence of inputs was then applied to the

\footnotetext{
${ }^{1}$ For Simulations 3 and 4 , see the provided video.
}

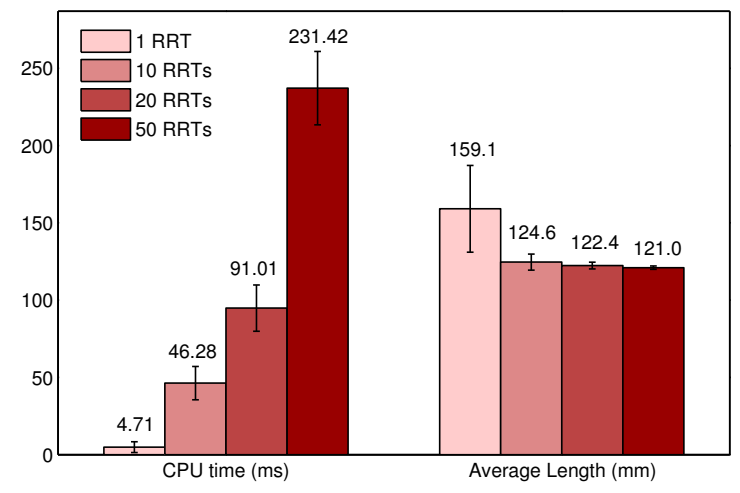

Fig. 4: Comparison of average path length and CPU time for different number of simultaneous trees in Simulation 2.

simulated needle and it resulted in the trajectory depicted in Fig. 5a. It can be observed that in the case of an ideal situation, the needle was able to follow the path almost exactly, with a small final error of $0.83 \mathrm{~mm}$ due to curvature discontinuity combined with discretization.

However, if we consider the presence of system uncertainties and perturbations such as positioning errors, tissue deformation and inhomogeneity, the result can greatly deviate from the expected. In simulation, these perturbations were modeled as white noises added to the measured tip configuration and to the actual natural curvature. The consequence of the addition of such noises can be seen in Fig. 5b, where the needle failed to avoid the obstacles and finished the insertion with $33.41 \mathrm{~mm}$ of error.

2) Simulation 4: The adaptive replanning strategy was evaluated at the same workspace and perturbation conditions as Simulation 3. In this last simulation, instead of applying the control inputs in open-loop, we used our adaptive strategy to systematically replan the trajectory along the insertion procedure. Fig. 5c illustrates how the online update of the path was able to compensate for the uncertainties, with a final error of only $0.20 \mathrm{~mm}$.

\section{CONCLUSIONS}

In this paper we proposed a closed-loop strategy for motion planning of steerable needles using duty-cycling and a new Arc-based RRT planner. The use of the duty-cycle technique instead of the usual stop-and-turn gave the system more trajectory possibilities. This multiplicity of solutions is specially useful for the case of steering around obstacles during insertion procedures restricted to a $2 \mathrm{D}$ working space.

The Arc-based RRT proved to respect the nonholonomic constraints while being fast enough to be used in an intraoperative system. We think it could be easily adapted to a preoperative planner by adding some new features, such as the calculation of initial orientation and insertion point and the possibility for the surgeon of choosing image regions as passage points for the needle.

The proposed replanning strategy made the insertion procedure more robust to system uncertainties such as tissue 


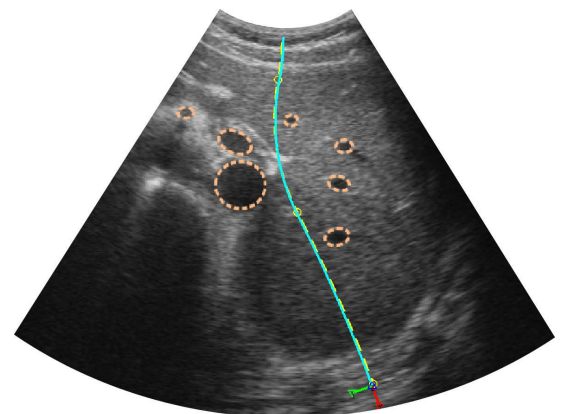

(a) Final error $=0.83 \mathrm{~mm}$

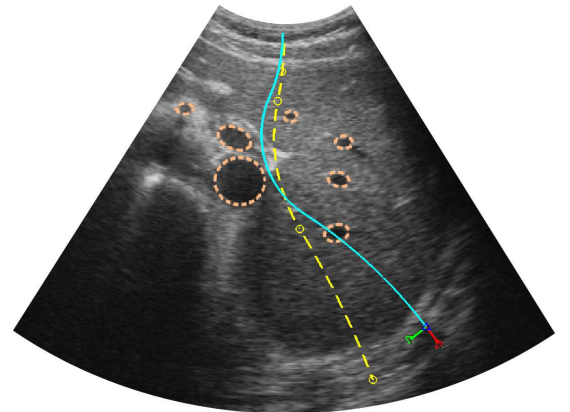

(b) Final error $=33.41 \mathrm{~mm}$

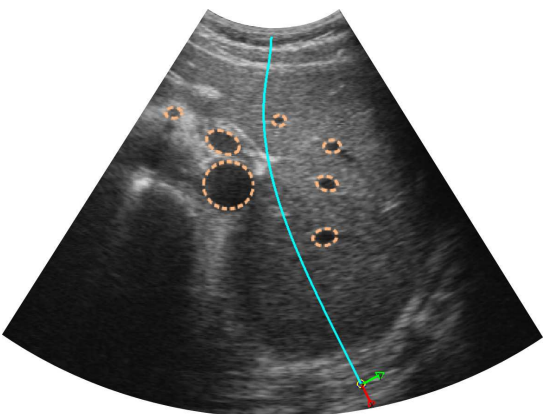

(c) Final error $=0.20 \mathrm{~mm}$

Fig. 5: Simulated trajectories of the needle tip in simulations of (a) an ideal situation, (b) under disturbances and (c) with adaptive replanning for noise compensation. Planned path (dashed) and simulated needle trajectory (solid).

deformation, errors in position, inhomogeneity and modeling approximations. The simulation results showed that even under perturbations, the needle was able to reach the target with satisfactory precision while an open-loop strategy would fail to avoid the obstacles and to arrive at the desired position. Preliminary work suggests that the replanning could also be used to compensate for small magnitude physiological movement if the tissue-needle combination presents good steerability. However, this possibility needs to be further investigated.

The next step is to evaluate the proposal on tissue phantoms and robotic hardware. We also plan to extend this method to 3D motion planning using the duty-cycle strategy for different planes in a tridimensional workspace. Some very recent papers [20], [21] propose the use of other samplebased algorithms for flexible needle path planning. A proper comparison of our approach and these new methods should be presented in a next work. Possible future works include the investigation of motion planners that combine the dutycycling strategy with smoother trajectories such as splines curves.

\section{ACKNOWLEDGMENTS}

This work is supported by CAPES under grant BEX 0154/10-5 and by the ANR ContInt USComp project.

\section{REFERENCES}

[1] R. Webster III, J. Kim, N. Cowan, G. Chirikjian, and A. Okamura, "Nonholonomic modeling of needle steering," The Int. Journal of Robotics Research, vol. 25, no. 5-6, pp. 509-525, 2006.

[2] N. Abolhassani, R. Patel, and M. Moallem, "Needle insertion into soft tissue: A survey," Medical Engineering \& Physics, vol. 29, no. 4, pp. 413-431, 2007.

[3] P. Svestka and M. Overmars, "Coordinated motion planning for multiple car-like robots using probabilistic roadmaps," in Proc. IEEE Int. Conf. on Robotics and Automation, 1995, pp. 1631-1636.

[4] S. M. LaValle and J. J. Kuffner, "Randomized kinodynamic planning," in Proc. IEEE Int. Conf. on Robotics and Automation, 1999, pp. 473479.

[5] J. Kuffner and S. LaValle, "RRT-connect: An efficient approach to single-query path planning," in Proc. IEEE Int. Conf. on Robotics and Automation, 2000, pp. 995-1001.

[6] W. Park, J. Kim, Y. Zhou, N. Cowan, A. Okamura, and G. Chirikjian, "Diffusion-based motion planning for a nonholonomic flexible needle model," in Proc. IEEE Int. Conf. on Robotics and Automation, 2005, pp. 4611-4616.
[7] V. Duindam, R. Alterovitz, S. Sastry, and K. Goldberg, "Threedimensional Motion Planning Algorithms for Steerable Needles Using Inverse Kinematics," The Int. Journal of Robotics Research, vol. 29, no. 7, pp. 789-800, 2010.

[8] J. Xu, V. Duindam, R. Alterovitz, and K. Goldberg, "Motion planning for steerable needles in 3D environments with obstacles using rapidlyexploring Random Trees and backchaining," in Proc. IEEE Int. Conf. on Automation Science and Engineering, 2008, pp. 41-46.

[9] R. Alterovitz, K. Goldberg, and A. Okamura, "Planning for Steerable Bevel-tip Needle Insertion Through 2D Soft Tissue with Obstacles," in Proc. IEEE Int. Conf. on Robotics and Automation, 2005, pp. 16401645.

[10] L. Vancamberg, A. Sahbani, S. Muller, and G. Morel, "Needle Path Planning for Digital Breast Tomosynthesis Biopsy," in Proc. IEEE Int. Conf. on Robotics and Automation, 2010, pp. 2062-2067.

[11] R. Alterovitz, M. Branicky, and K. Goldberg, "Motion Planning Under Uncertainty for Image-Guided Medical Needle Steering," The Int. Journal of Robotics Research, vol. 27, no. 11-12, pp. 1361-1374, 2008.

[12] R. Alterovitz and K. Goldberg, "The Stochastic Motion Roadmap: A Sampling Framework for Planning with Markov Motion Uncertainty," Robotics: Science and Systems, 2007.

[13] K. Hauser, R. Alterovitz, N. Chentanez, A. Okamura, and K. Goldberg, "Feedback Control for Steering Needles Through 3D Deformable Tissue Using Helical Paths," in Proc. Robots: Science and Systems, 2009.

[14] R. Alterovitz, A. Lim, K. Goldberg, G. Chirikjian, and A. Okamura, "Steering Flexible Needles Under Markov Motion Uncertainty," in Proc. IEEE/RSJ Int. Conf. on Intelligent Robots and Systems, 2005, pp. $1570-1575$.

[15] J. Engh, G. Podnar, S. Khoo, and C. Riviere, "Flexible Needle Steering System for Percutaneous Access to Deep Zones of the Brain," in Proc. IEEE Annual Northeast Bioengineering Conf., 2006, pp. 103-104.

[16] N. Abolhassani, R. Patel, and M. Moallem, "Experimental study of robotic needle insertion in soft tissue," in Proc. Int. Congress and Exhibition on Computer Assisted Radiology and Surgery, vol. 1268, 2004, pp. 797-802.

[17] D. Minhas, J. Engh, M. Fenske, and C. Riviere, "Modeling of needle steering via duty-cycled spinning," in Proc. Annual Int. Conf. of the IEEE Eng. in Medicine and Biology Society, 2007, pp. 2756-2759.

[18] B. Adorno, P. Fraisse, and S. Druon, "Dual position control strategies using the cooperative dual task-space framework," in IEEE/RSJ Int. Conf. on Intelligent Robots and Systems, 2010, pp. 3955-3960.

[19] V. Kallem and N. Cowan, "Image Guidance of Flexible Tip-Steerable Needles," IEEE Trans. on Robotics, vol. 25, no. 1, pp. 191-196, 2009.

[20] R. Alterovitz, S. Patil, and A. Derbakova, "Rapidly-Exploring Roadmaps: Weighing Exploration vs. Refinement in Optimal Motion Planning," in Proc. IEEE Int. Conf. on Robotics and Automation, 2011, pp. 3706-3712.

[21] E. Lobaton, J. Zhang, S. Patil, and R. Alterovitz, "Planning CurvatureConstrained Paths to Multiple Goals Using Circle Sampling," in Proc. IEEE Int. Conf. on Robotics and Automation, 2011, pp. 1463-1469. 